\title{
Molecular modelling studies to suggest novel scaffolds against SARS-CoV-2 target enzymes
}

\author{
Ahmet Fatih ŞAHİN 1 (D) , Ş. Güniz KÜÇÜKGÜZEL 2 (D), Atilla AKDEMİR 1,3* (D) \\ 1 Department of Drug Discovery and Development, Institute of Health Sciences, Bezmialem Vakif University, 34093 \\ Fatih, İstanbul, Turkey. \\ 2 Vocational School of Health Services, Fenerbahçe University, 34758, Ataşehir, Istanbul, Turkey. \\ 3 Computer-aided Drug Discovery Laboratory, Department of Pharmacology, Faculty of Pharmacy, Bezmialem Vakif \\ University, 34093, Istanbul, Turkey. \\ * Corresponding Author. E-mail: aakdemir@bezmialem.edu.tr (A.A.); Tel. +90 2125232288 Ext. 3142
}

Received: 12 July 2021 / Revised: 20 October 2021 / Accepted: 23 October 2021

\begin{abstract}
In this study, molecular modelling study of previously synthesized compounds against SARS-CoV-2 target enzyme was performed. A subset of 156 compounds from an in-house database has been subjected to molecular modelling studies against the SARS-CoV-2 ADP-ribose phosphatase (ADRP, NSP3), Papain-like protease (PLpro), and uridine specific endoribonuclease (NSP15) enzymes. We have identified one compound that is expected to inhibit the SARS-CoV-2 ADRP enzyme and one compound that is expected to inhibit the NSP15 enzyme.
\end{abstract}

KEYWORDS: SARS-CoV-2; Covid-19; ADRP; PLpro; Nsp15; docking; molecular dynamics.

\section{INTRODUCTION}

The novel coronavirus (SARS-CoV-2) is a respiratory tract infection that was first detected in Wuhan, China [1]. This novel virus causes the severe acute respiratory disease named Coronavirus Disease 2019 (COVID-19). Covid-19 is highly transmissible and has spread fast all over the world. This disease shows itself with symptoms such as high fever, cough, shortness of breath, headache, sore throat, runny nose, muscle and joint pain, weakness, loss of sense of smell and taste, and diarrhoea.

The SARS-CoV-2 is an enveloped single stranded RNA virus that contains four structural proteins, namely Spike (S), Envelope (E), Membrane (M), and Nucleocapsid (N) [2-4]. The S protein consists of the S1 and S2 subunits. The S1 subunit is involved in the ACE2 mediated virus attachment, while the S2 subunit provides membrane fusion. The $\mathrm{N}$ protein also plays an important role in viral entry. The $\mathrm{E}$ protein is necessary for viral assembly, and the $\mathrm{M}$ protein promotes spike incorporations as well as the facilitation of virion production [2]. In addition, the SARS-CoV-2 virus contains 15 non-structural proteins (NSP 1-15). Each NSP has a role in the life cycle and pathogenicity of the virus [5]. Here we focus on ADP-ribose phosphatase (ADRP, NSP3), papain-like protease (PLpro) and a uridine-specific endoribonuclease (NSP15).

ADRP is responsible for both induction of viral replication as well as interference with the host immune response [6, 7]. Papain-like protease (PLpro) is one of the major important cysteine proteases of SARS-CoV-2 that processes the polyproteins translated from the viral RNA-genome to yield the active functional proteins necessary for viral replication [8]. Some studies shows that a coronavirus endoribonuclease delays activation of the host sensor system. The uridine-specific endoribonuclease is present in all coronaviruses. It processes viral RNA to avoid detection by RNA-activated host defence systems. Therefore, it considers as a promising drug target $[9,10]$.

This manuscript describes molecular modelling studies of 156 previously synthesized [11-20] and in house available compounds against the Sars-CoV-2 ADP-ribose phosphatase (ADRP, NSP3), Papain-like protease (PLpro, NSPX), and uridine specific endoribonuclease (NSP15) enzymes. Our results suggest that 2 compounds not previously investigated as inhibitors of Sars-CoV-2 enzymes may inhibit ADRP.

How to cite this article: Şahin AF, Küçükgüzel ŞG, Akdemir A. Molecular modelling studies to suggest novel scaffolds against SARS-CoV-2 target enzymes. J Res Pharm. 2021; 25(6): 1010-1017. 


\section{RESULTS AND DISCUSSION}

Molecular modelling studies were performed to investigate whether the compounds under investigation could interact with the SARS-CoV-2 target proteins ADRP, PLpro, and uridine specific endoribonuclease. To this end, the compounds were docked into the active sites of the target enzymes. The highest scoring docked poses that showed complementarity with the active site were selected for $50 \mathrm{~ns}$ molecular dynamics (MD) simulations to investigate the stability of the pose, the dynamic binding interactions, and the binding energy.

\subsection{Modelling studies against ADRP}

All compounds were docked into the ADRP structure in the complex with ADP ribose (pdb: 6W02, 1.50 $\AA$ A). Compounds 1 and $\mathbf{2}$ showed docked poses that suggested binding to the active site of ADRP (Figure 1).
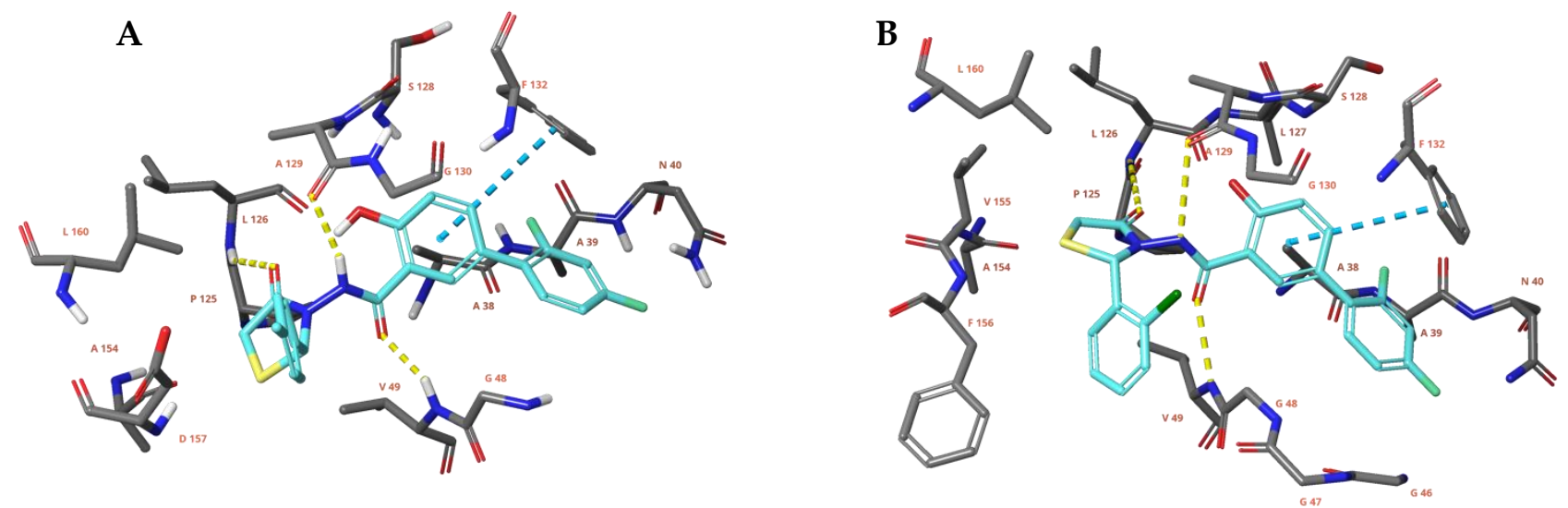

Figure 1. The docked poses of $\mathbf{1}$ (A), 2 (B) in the active site of ADRP (pdb: 6W02).

The docked pose of 1 [13] forms hydrogen bonds with the backbones of Val49, Leu126 and Ala129 (Figure 1A). In addition, the ligand forms $\pi$ - $\pi$ stacking with the sidechain of Phe132. The docked pose of $\mathbf{1}$ was stable during a $50 \mathrm{~ns}$ molecular dynamics simulation. The hydrogen bond between the ligand and the backbones of Val49, Leu126 and Ala129 were observed during $41 \%, 67 \%$ and $62 \%$ of the simulation time, respectively (Figure 2A). In addition, the ligand formed an interaction with Leu126 via a bridging water molecule. The $\pi-\pi$ stacking with the sidechain of Phe132 was present during $35 \%$ of the simulation. Hydrophobic interactions occurred with Ala38 and Ile131. The calculated binding energy fluctuates between approximately $-70 \mathrm{kcal} / \mathrm{mol}$ and $-50 \mathrm{kcal} / \mathrm{mol}$ during the simulation (Figure $2 \mathrm{~B}$ ).

A

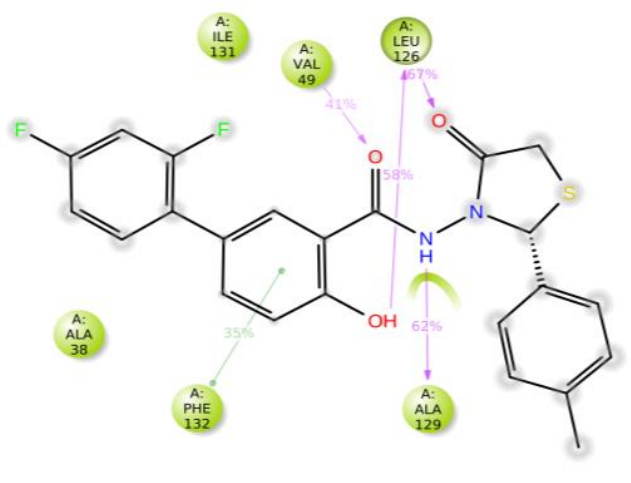

B

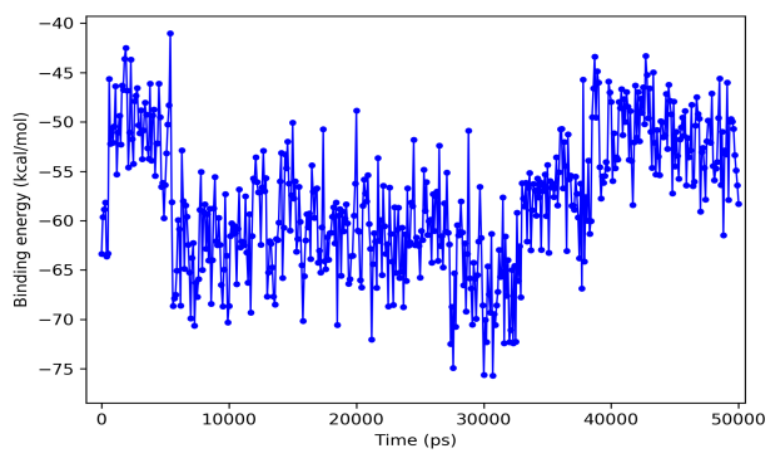

Figure 2. A) The binding interactions of 1 with the active site of ADRP during a $50 \mathrm{~ns}$ MD simulation. B) The MM-GBSA binding energy. Hydrophobic amino acids are indicated in green. Hydrogen bonds are indicated in purple and п-п stacking in green. Solvent accessible ligand atoms are indicated with a grey shading. The contact surface with hydrophobic residues in indicated in green. 
The docked pose of $\mathbf{2}$ [16] in the active site of ADRP shows that oxygen of thiazolidinone of the ligand forms hydrogen bond with backbone nitrogen of Leu126. The backbone oxygen of Ala129 forms hydrogen bond with nitrogen of the ligand. In addition, other oxygen of the ligand forms hydrogen bond with the backbone nitrogen of the ligand and phenol ring of the ligand forms $\pi-\pi$ stacking with Phe132 (Figure 1B). The docked pose of 2 was selected for a 50 ns MD simulation.

During a $50 \mathrm{~ns}$ molecular dynamics simulation the docked pose of 2 was stable. Hydrogen bonds with Leu126 and Ala129 were observed during \%64 and \%97 of the simulation time, respectively. In addition, an interaction via bridging water molecules occurs between the ligand's oxygen and Ile131 during \%63 of simulation time. The hydrogen bond which has been seen on docked pose interactions occurs during $\% 13$ of simulation time and have interactions occasionally via bridging water molecule between the ligand's two oxygen atoms and Val49 ( $<20 \%$ of the simulation time). Additionally, hydrogen bonds occur with Ser128 and Phe132 during \%11 and \%12 of the simulation time respectively (Figure 3A). The calculated binding energy fluctuates around approximately $-75 \mathrm{kcal} / \mathrm{mol}$ during the simulation (Figure 3B).
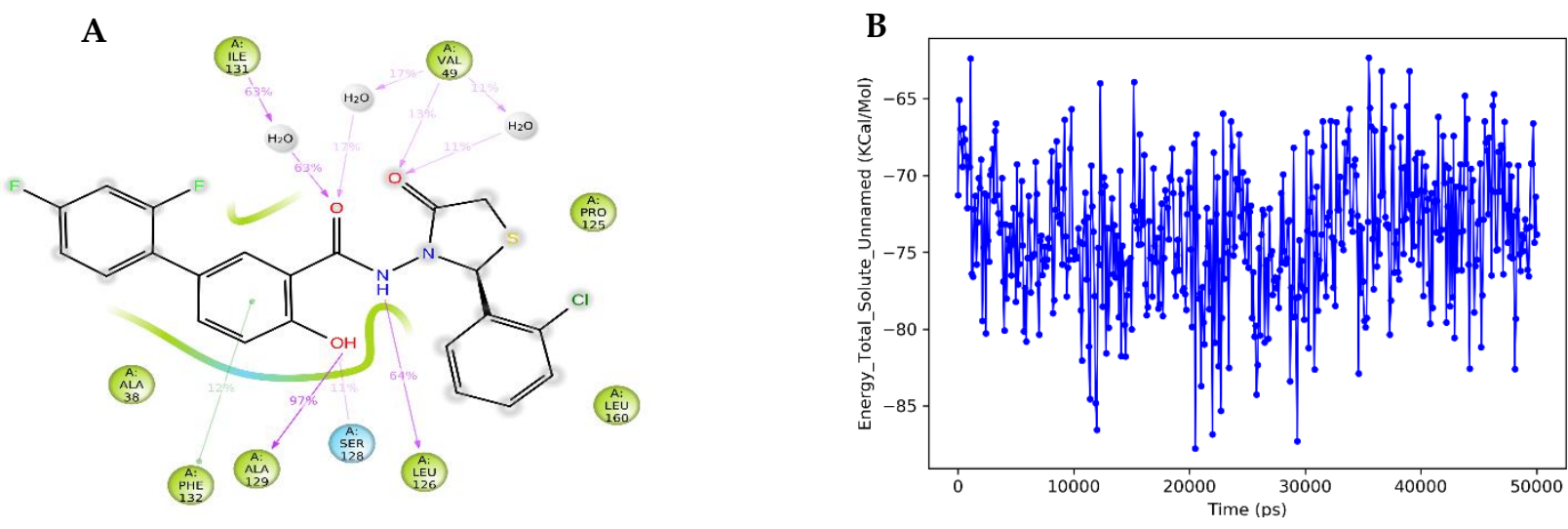

Figure 3. A) The binding interactions of 2 with the active site of ADRP during a 50 ns MD simulation. B) The MMGBSA binding energy. Hydrophobic amino acids are indicated in green and cationic residues are indicated in red. Hydrogen bonds are indicated in purple. Solvent accessible ligand atoms are indicated with a grey shading. The contact surface with cationic residues in indicated in red.

The cocrystal structure of APR in the active site of ADRP reveals that the APR's adenosine group form hydrogen bonds with the sidechain of Asp22 and the backbone of Ile23 (Figure S1, A). The ligand's carbonyl and phosphate groups form hydrogen bonds with the backbone nitrogen of Val49, Ser128 and Ile131. The ribose moiety forms hydrogen bonds with the backbone nitrogen of Asn40 and Gly48 and an aromatic hydrogen bond with the sidechain of Phe132. Hydrogen bonds with the ribose moiety of ligand is expected to be stronger compared to the aromatic hydrogen bond.

During a $50 \mathrm{~ns}$ molecular dynamics simulation the binding pose as observed in the cocrystal structure was stable. The hydrogen bonds with Asp22 and Ile123 were observed during 99\% and 98\% of the simulation time respectively. Hydrogen bond with Val49 was observed during $77 \%$ of the simulation period. In addition, interactions via bridging water molecules occurs between the ligand's phosphate and Ala38 and Ala50 during $\% 82$ and $\% 84$ of the MD simulation time. Furthermore, other several interactions via bridging water molecules occurs between ligand and active site during \%30 - \%55 of the MD simulation (Figure S1, B).

The calculated binding energy increases from approximately $-100 / 90 \mathrm{kcal} / \mathrm{mol}$ to approximately $-80 /-70$ $\mathrm{kcal} / \mathrm{mol}$ during the simulation (Figure S1, C).

\subsection{Modelling studies against PLpro}

Only compounds 3 and 4 [16] were candidates for MD simulation. The docked pose of 3 in the active site of PLpro shows that hydrogen bonds are formed with Asp164, Arg166 and Tyr268 (Figure 4A). In addition, $\pi-\pi$ stackings are formed with the sidechains of Tyr264 and Tyr268. The docked pose of 4 indicates the presence of hydrogen bonds with Tyr264 and Tyr273 (Figure 4B). Both poses were subjected to $50 \mathrm{~ns}$ MD simulations and neither the binding interactions nor the binding energy suggested binding of the compounds to PLpro. 

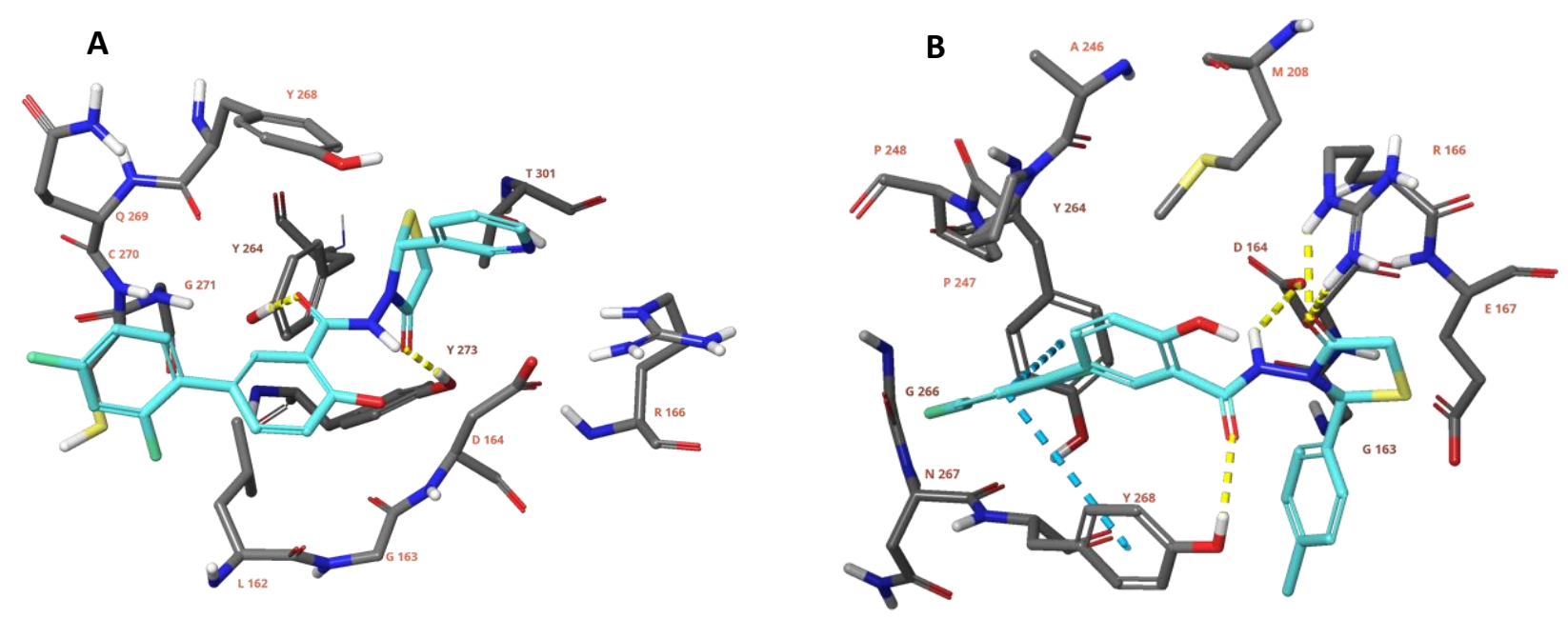

Figure 4. The docked poses of 3 (A) and 4 (B) in the active site of PLpro (pdb: 6W9C).

\subsection{Modelling studies against uridin specific endoribonuclease (NSP15)}

All compounds were docked into the active site of NSP15 and four docked poses were subjected to MD analysis. The docked pose of 5 [14] shows that ligand's two nitrogen atoms form hydrogen bonds with the sidechain and backbone of Ser294. The other nitrogen atom of the ligand forms a hydrogen bond with the sidechain of Lys290. Additionally, the oxygen atom of the ligand forms a hydrogen bond with the backbone of Gly248 (Figure 5A). Analysis of the MD trajectory indicates that the docked pose is not stable, and the binding energy increases towards approximately $-30 \mathrm{kcal} / \mathrm{mol}$ (Figure 6).

The docked pose of 6 [14] shows the presence of a hydrogen bond with Ser294 and $\pi-\pi$ stacking with Tyr343. (Figure 5B). Again, the MD simulations indicate that the poses are not stable, and the binding energies do not suggest strong binding (Figure 7).

Finally, the docked pose of 7 [15] shows the presence of hydrogen bonding interactions with the sidechain of Lys250 and the backbones of Gly248 and Ser294 (Figure 5C). In addition, $\pi-\pi$ stackings with the sidechains of His235 and Trp333 are formed. During the MD simulation these interactions remained stable during the simulation; i.e. Gly248 (99\%), Lys250 (79\%), Ser294 (95\%), His235 (93\%) and Trp333 (44\%) (Figure $8 \mathrm{~A})$. The binding energy was reasonably stable in the $-60 /-50 \mathrm{kcal} / \mathrm{mol}$ in the final period of the simulation (Figure 8B).

\section{CONCLUSION}

Previously synthesized compounds were subjected to molecular modelling studies, which consists of docking studies and molecular dynamics simulations, to investigate their potential to inhibit SARS-CoV-2 target enzymes. The results suggest that one compound (i.e., 2) may be able to inhibit ADRP and one compound (compound 7) NSP15.

\section{MATERIALS AND METHODS}

\subsection{Preparation of protein structures}

The Crystal structure of Sars-CoV-2 ADP ribose phosphatase (ADRP, NSP3, pdb: 6W02), Papain-like protease (PLpro, pdb: 6W9C) and uridine specific endoribonuclease (NSP15, pdb: 6WLC) were obtained from the RCSB Protein Data Bank. Subsequently, the structure was prepared using the protein preparation tool of Schrödinger (v2021-1, Schrödinger, Inc., New York, USA). All water and buffer molecules were omitted. Subunit A was retained and all other subunits, if present, were omitted. Subsequently, hydrogen atoms were added, and the system was minimized using the OPLS4 forcefield. 
A

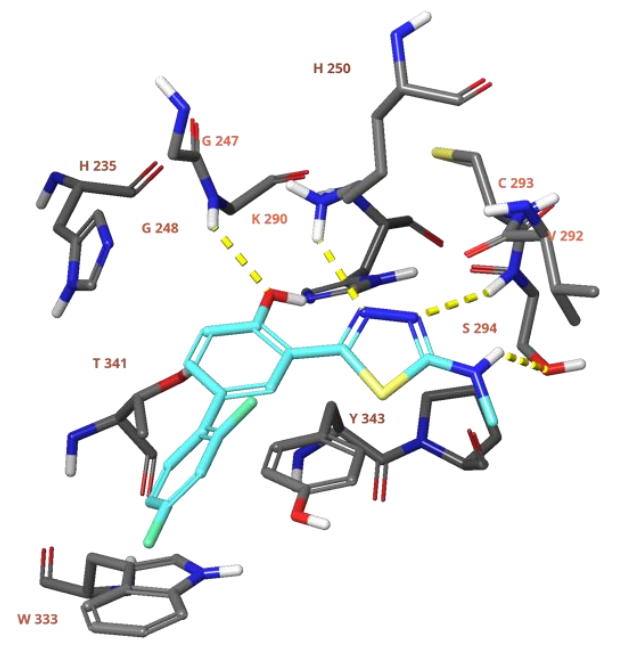

B

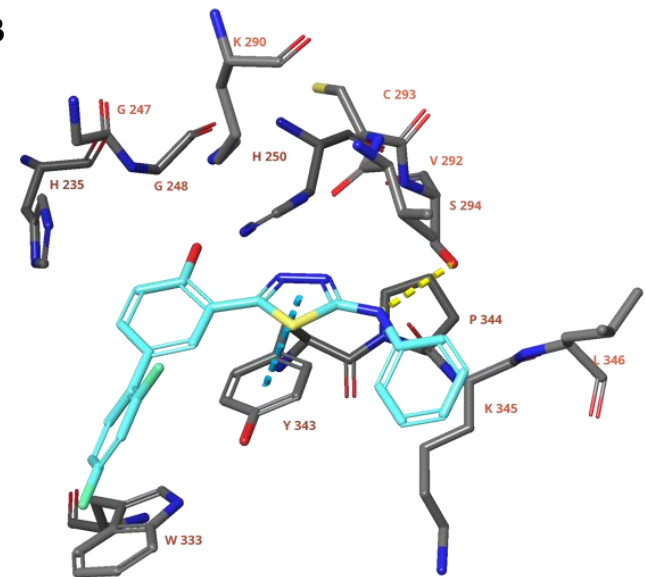

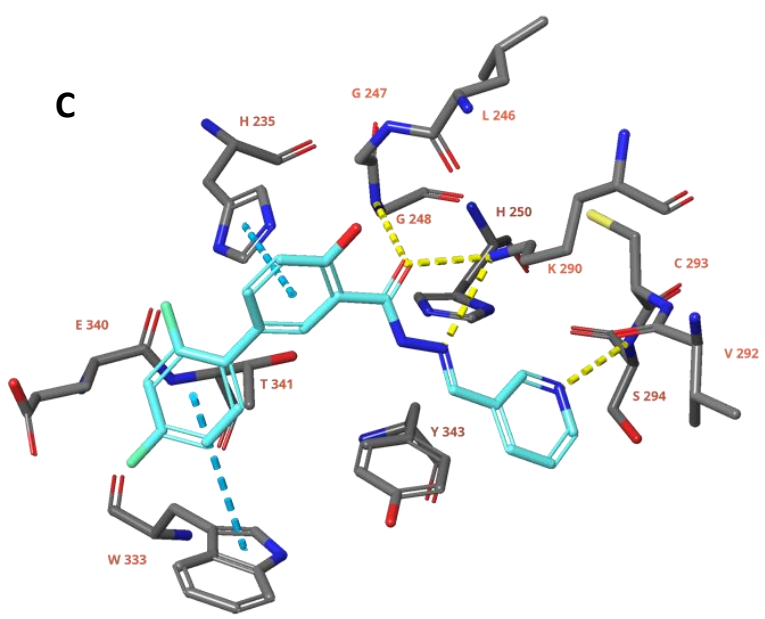

Figure 5. The docked poses of 5 (A), 6 (B) and 7 (C) in the active site of NSP15 (pdb: 6WLC).

A

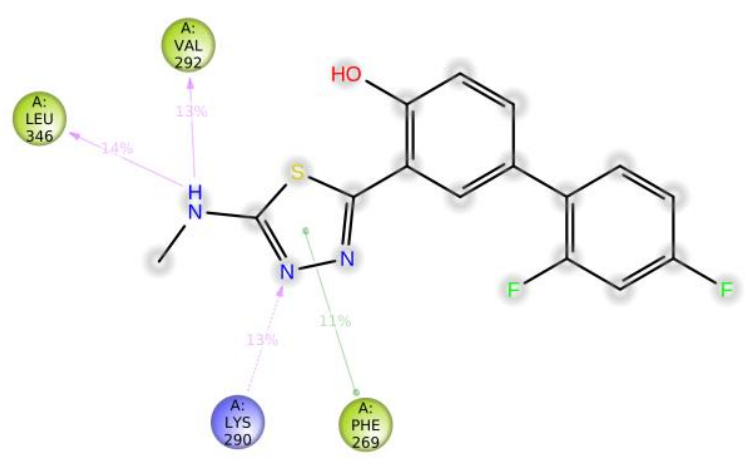

B

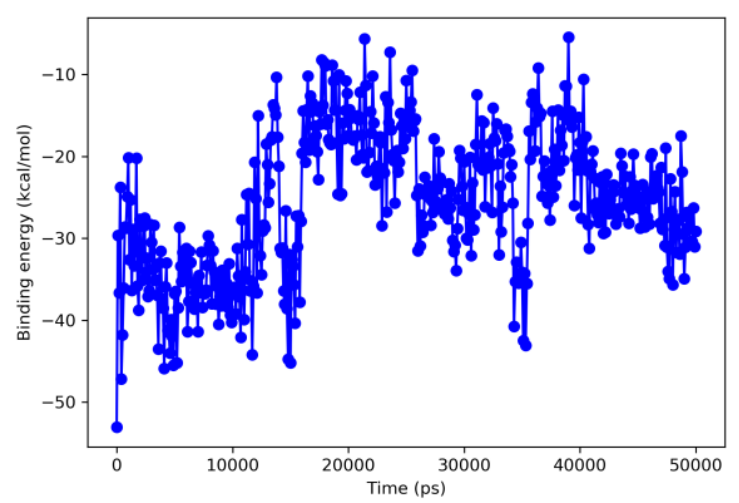

Figure 6. A) The binding interactions of 5 with the active site of NSP15 during a 50 ns MD simulation. B) The MM-GBSA binding energy. Hydrophobic amino acids are indicated in green and cationic residues are indicated in purple. Hydrogen bonds and $\pi-\pi$ stackings are indicated in purple and green respectively. Solvent accessible ligand atoms are indicated with a grey shading. The contact surface with cationic residues in indicated in red. 

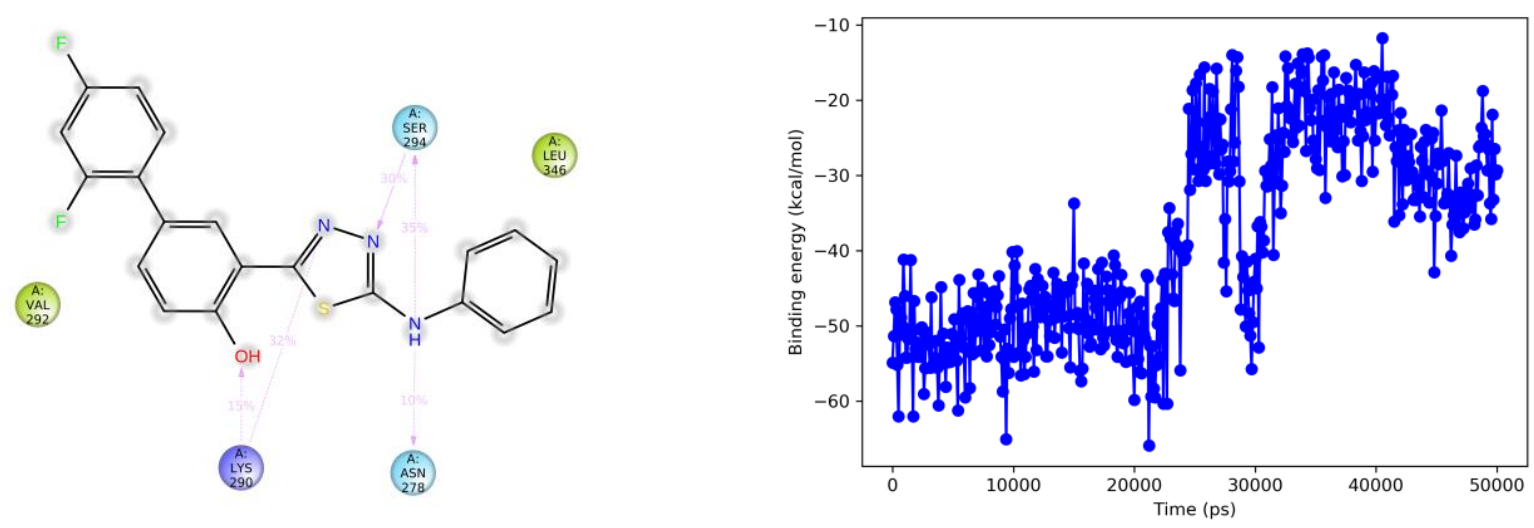

Figure 7. A) The binding interactions of 6 with the active site of NSP15 during a 50 ns MD simulation. B) The MM-GBSA binding energy. Hydrophobic amino acids are indicated in green and cationic residues are indicated in purple. Hydrogen bonds and $\pi-\pi$ stackings are indicated in purple and green respectively. Solvent accessible ligand atoms are indicated with a grey shading. The contact surface with cationic residues in indicated in red.

A<smiles>CC(=NNC(=O)c1cc(-c2ccc(F)cc2F)cc(Br)c1OB(O)c1ccccc1)c1cccnn1</smiles>

B

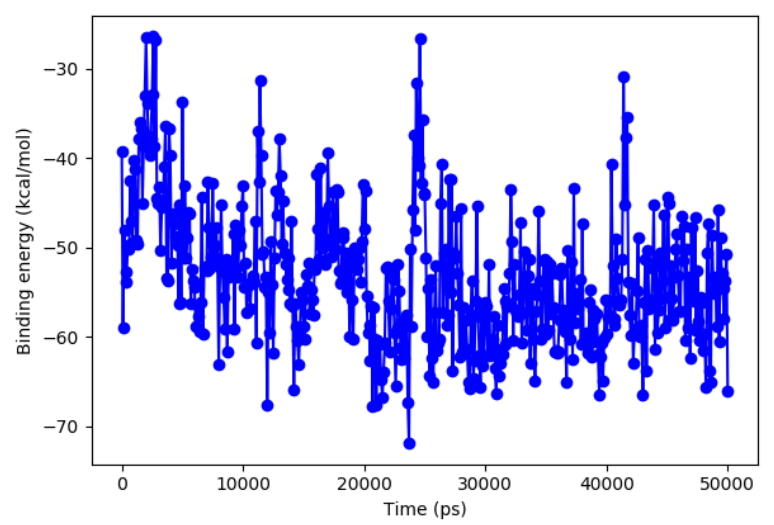

Figure 8. A) The binding interactions of 7 with the active site of NSP15 during a 50 ns MD simulation. B) The MM-GBSA binding energy. Hydrophobic amino acids are indicated in green, polar residues are indicated in blue and cationic residues are indicated in red. Hydrogen bonds and $\pi-\pi$ stackings are indicated in purple and green respectively. Solvent accessible ligand atoms are indicated with a grey shading. The contact surface with cationic residues in indicated in red.

\subsection{Docking Studies}

The ligand set was prepared using the LigPrep tool of Schrödinger and minimized with the OPLS4 forcefield. Subsequently, all ligands were docked into the binding sites of the target enzymes. The binding sites have been assigned as all residues within $5 \AA$ of the cocrystallized ligand. Docking was performed using the Glide tool of Schrödinger with the SP settings. The three highest scoring poses were obtained for each ligand and the poses were subsequently minimized using the Prime tool and MM-GBSA forcefield. To this end, the ligand and all residues within $5 \AA$ were unrestrained.

High scoring compounds that formed binding interactions (hydrogen bonds, electrostatic interactions, and hydrophobic interactions) and showed complementarity in shape and (a)polarity were selected for molecular dynamics (MD) simulations. 


\subsection{Molecular dynamics simulations}

The ligand-enzyme complexes obtained with the docking procedure were subjected to a $50 \mathrm{~ns} \mathrm{MD}$

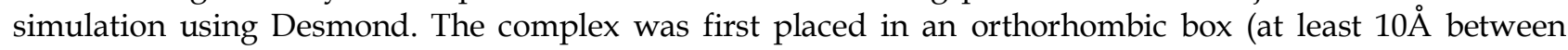
complex and boundary) and then filled with Tip5P water molecules and $0.15 \mathrm{M} \mathrm{NaCl}$. The amount of $\mathrm{Na}^{+}$or $\mathrm{Cl}$ - ions were adjusted to create a neutral system. Afterwards, all heavy atoms were restrained, and the system was minimized for 100 ps using the OPLS4 forcefield. Finally, the system was simulated for 50 ns under isothermic (Nose-Hoover chain, 1ps relaxation time) and isobaric (Martyna-Tobial-Klein, 2ps relaxation time, isotropic coupling) conditions without restraints. Snapshots were saved every 100 ps. Finally, the ligandprotein binding interactions as well as the MM-GBSA binding energy were calculated.

Author contributions: Concept - Ş.G.K., A.A.; Design - A.A.; Supervision - A.A.; Data Collection and/or Processing A.F.Ş.; Analysis and/or Interpretation - A.F.Ş., A.A.; Literature Search - A.F.Ş., Ş.G.K.; Writing - A.F.Ş., A.A.; Critical Review - A.F.Ş., Ş.G.K., A.A.

Conflict of interest statement: The authors declared no conflict of interest.

Appendix A. Supplementary Material

Supplementary material related to this article can be accessed at https://dx.doi.org/10.29228/jrp.96 .

\section{REFERENCES}

[1] Hu B, Guo H, Zhou P, Shi ZL. Characteristics of SARS-CoV-2 and COVID-19. Nat Rev Microbiol. 2021; 19(3): 141154. [CrossRef]

[2] Satarker S, Nampoothiri M. Structural Proteins in Severe Acute Respiratory Syndrome Coronavirus-2. Arch Med Res. 2020; 51(6): 482-491. [CrossRef]

[3] Gordon DE, Jang GM, Bouhaddou M, et al. A SARS-CoV-2 protein interaction map reveals targets for drug repurposing. Nature. 2020; 583(7816): 459-468. [CrossRef]

[4] Prajapat M, Sarma P, Shekhar N, Avti P, Sinha S, Kaur H, Kumar S, Bhattacharyya A, Kumar H, Bansal S, Medhi B.. Drug targets for corona virus: A systematic review. Indian J Pharmacol. 2020; 52(1): 56-65. [CrossRef]

[5] Raj R. Analysis of non-structural proteins, NSPs of SARS-CoV-2 as targets for computational drug designing. Biochem Biophys Rep. 2020; 25: 100847. [CrossRef]

[6] Michalska K, Kim Y, Jedrzejczak R, Maltseva NI, Stols L, Endres M, Joachimiak A.Crystal structures of SARS-CoV-2 ADP-ribose phosphatase: from the apo form to ligand complexes. IUCrJ. 2020; 7(Pt 5): 814-824. [CrossRef]

[7] Xu Y, Cong L, Chen C, Wei L, Zhao Q, Xu X, Ma Y, Bartlam M, Rao Z. Crystal structures of two coronavirus ADPribose-1"-monophosphatases and their complexes with ADP-Ribose: a systematic structural analysis of the viral ADRP domain. J Virol. 2009; 83(2): 1083-1092. [CrossRef]

[8] Rajpoot S, Alagumuthu M, Baig MS. Dual targeting of 3CLpro and PLpro of SARS-CoV-2: A novel structurebased design approach to treat COVID-19. Curr Res Struct Biol. 2021; 3: 9-18. [CrossRef]

[9] Pillon MC, Frazier MN, Dillard LB, Williams JG, Kocaman S, Krahn JM, Perera L, Hayne CK, Gordon J, Stewart ZD, Sobhany M, Deterding LJ, Hsu AL, Dandey VP, Borgnia MJ, Stanley RE. Cryo-EM structures of the SARS-CoV-2 endoribonuclease Nsp15 reveal insight into nuclease specificity and dynamics. Nat Commun. 2021; 12(1): 636. [CrossRef]

[10] Hackbart M, Deng X, Baker SC. Coronavirus endoribonuclease targets viral polyuridine sequences to evade activating host sensors. Proc Natl Acad Sci USA. 2020; 117(14): 8094-8103. [CrossRef]

[11] Aydın S, Kaushik-Basu N, Arora P, Basu A, Brian Nichols D, T Talele T, Akkurt M, Çelik İ, Büyükgüngör O, Küçükgüzel ŞG. Microwave assisted synthesis of some novel Flurbiprofen hydrazidehydrazones as anti-HCV NS5B and anticancer agents. Marmara Pharm J. 2013; 17(1): 26-34. [CrossRef]

[12] Aydın S, Kaushik-Basu N, Ozbas-Turan S, Akbuga J, Mega-Tiber P, Orun O, Gurukumar KR, Basu A, Küçükgüzel ŞG. Synthesis of 1-aroyl-3,5-dimethyl-1H-pyrazoles as anti-HCV and anticancer agents. Lett Drug Des Discov. 2014; 11(2): 121-131. [CrossRef]

[13] Küçükgüzel G, Kocatepe A, De Clercq E, Sahin F, Güllüce M. Synthesis and biological activity of 4-thiazolidinones, thiosemicarbazides derived from diflunisal hydrazide. Eur J Med Chem. 2006; 41(3): 353-359. [CrossRef] 
[14] Küçükgüzel SG, Küçükgüzel I, Tatar E, Rollas S, Sahin F, Güllüce M, De Clercq E, Kabasakal L. Synthesis of some novel heterocyclic compounds derived from diflunisal hydrazide as potential anti-infective and anti-inflammatory agents. Eur J Med Chem. 2007; 42(7): 893-901. [CrossRef]

[15] Şenkardeş S, Kaushik-Basu N, Durmaz İ, Manvar D, Basu A, Atalay R, Küçükgüzel ŞG. Synthesis of novel diflunisal hydrazide-hydrazones as anti-hepatitis $\mathrm{C}$ virus agents and hepatocellular carcinoma inhibitors. Eur J Med Chem. 2016;108: 301-308. [CrossRef]

[16] Şenkardeş S, Özakpınar ÖB, Özsavcı D, Şener A, Çevik Ö, Küçükgüzel ŞG. Synthesis of diflunisal thiazolidinones as anticancer agents. Anticancer Agents Med Chem. 2016;16(10): 1266-1274. [CrossRef]

[17] Karakuş S, Küçükgüzel ŞG, Küçükgüzel I, De Clercq E, Pannecouque C, Andrei G, Snoeck R, Sahin F, Bayrak OF. Synthesis, antiviral and anticancer activity of some novel thioureas derived from N-(4-nitro-2-phenoxyphenyl)methanesulfonamide. Eur J Med Chem. 2009;44(9):3591-3595. [CrossRef]

[18] Küçükgüzel ŞG, Coşkun İ, Aydın S, Aktay G, Gürsoy Ş, Çevik Ö, Özakpınar ÖB, Özsavcı D, Şener A, Kaushik-Basu N, Basu A, Talele TT. Synthesis and characterization of celecoxib derivatives as possible anti-inflammatory, analgesic, antioxidant, anticancer and anti-HCV agents. Molecules. 2013;18(3):3595-3614. [CrossRef]

[19] Coşkun GP, Djikic T, Hayal TB, Türkel N, Yelekçi K, Şahin F, Küçükgüzel ŞG. Synthesis, Molecular Docking and Anticancer Activity of Diflunisal Derivatives as Cyclooxygenase Enzyme Inhibitors. Molecules. 2018;23(8):1969. [CrossRef]

[20] Coşkun GP, Djikic T, Kalaycı S, Yelekçi K, Şahin F, Küçükgüzel ŞG. Synthesis, Molecular Modelling and antibacterial activity against Helicobacter pylori of novel diflunisal derivatives as urease enzyme inhibitors. Lett Drug Des Discov. 2019;16(4): 392-400. [CrossRef] 